\title{
NOTA SOBRE LA PRESENCIA DE SPARTINA ALTERNIFLORA LOISEL. (POACEAE: CHLORIDOIDEAE: CYNODONTEAE) EN HUMEDALES MEXICANOS
}

\author{
Arturo Mora-Olivo ${ }^{1 *}$ y Jesús Valdés-Reyna ${ }^{2}$ \\ ${ }^{1}$ Universidad Autónoma de Tamaulipas, Instituto de Ecología Aplicada, División \\ del Golfo núm. 356, Colonia Libertad, 87019 Cd. Victoria, Tamaulipas, México \\ ${ }^{2}$ Universidad Autónoma Agraria Antonio Narro, Departamento de Botánica, \\ Colonia Buenavista, 25315 Saltillo, Coahuila, México, jvaldes@uaaan.mx
}

\section{RESUMEN}

Se reporta la presencia de Spartina alterniflora Loisel. para México. Esta gramínea acuática se registró en estuarios de los estados de Tamaulipas y Veracruz durante un estudio de la flora asociada a humedales mexicanos. Se proporciona una clave para la identificación de los componentes mexicanos de Spartina, la descripción de la especie e información sobre aspectos ecológicos básicos.

Palabras clave: México, planta acuática, Spartina, Tamaulipas, Veracruz.

\begin{abstract}
The presence of Spartina alterniflora Loisel. for Mexico is reported. This aquatic grass was registered in estuaries of the states of Tamaulipas and Veracruz during a study of the flora associated with Mexican wetlands. A taxonomic key, the description of the species and information on basic environmental aspects is provided.
\end{abstract}

Key words: aquatic plant, Mexico, Spartina, Tamaulipas, Veracruz.

\footnotetext{
*Autor para correspondencia: amorao@uat.edu.mx
} 
Recientemente, durante el estudio de la flora acuática de México, se registró una gramínea acuática del género Spartina Schreb. para los estados de Tamaulipas y Veracruz, para lo cual se procedió a su identificación.

A nivel mundial existen 17 especies de Spartina (Clayton et al., 2009) y de acuerdo con Dávila et al. (2006) en México sólo se presentan cinco (S. cynosuroides (L.) Roth, S. foliosa Trin., S. gracilis Trin., S. patens (Aiton) Muhl. y S. spartinae (Trin.) Merr. ex Hitchc.). Durante la revisión de los ejemplares botánicos de este género en herbarios nacionales (ENCB, MEXU, UAT, XAL) se encontró que la cita de Spartina alterniflora Loisel., por Espejo-Serna y colaboradores (2000) para los estados de Chihuahua, Coahuila y Tamaulipas se basó en material que en realidad corresponde a otras especies como Distichlis spicata y Spartina spartinae.

Después de una minuciosa identificación por medio de las claves y revisión de ejemplares de varios herbarios, la gramínea acuática que se colectó fue identificada como Spartina alterniflora Loisel., por lo cual su presencia en México se confirma con fundamento en ejemplares colectados en el Golfo de México en los estados de Tamaulipas y Veracruz. Con ésta cabe reconocer que el género Spartina en México está representado por seis especies.

Clave para la identificación de las especies del género Spartina de México (Modificada de Mobberley, 1956 y de Barkworth, 2003).

1. Plantas sin rizomas, culmos cespitosos formando grandes macollos S. spartinae

1. Plantas rizomatosas, culmos solitarios o formando pequeños macollos 2

2. Márgenes de las láminas fuertemente escabrosos 3

3. Láminas de 2.5-8 mm de ancho, quilla de la gluma superior conspicuamente pilosa; anteras dehiscentes en la madurez S. gracilis

3. Láminas de $10-25 \mathrm{~mm}$ de ancho, quilla de la gluma superior híspida; anteras indehiscentes en la madurez S. cynosuroides

2. Márgenes de las láminas glabros o ligeramente escabrosos 4

4. Culmos no carnosos; láminas involutas, de 1-4 mm de ancho; lígulas de $0.5(-1) \mathrm{mm}$ de largo S. patens

4. Culmos carnosos o suculentos; láminas extendidas o ligeramente involutas, de 3-25 mm de ancho; lígulas de 1-2 mm de largo .......................... 5

5. Rizomas y escamas sin tejido aerenquimatoso; lemas con ápice obtuso, redondeado o lobado; plantas nativas de la costa del océano Pacífico (California y Baja California) S. foliosa 
Mora-Olivo y Valdés-Reyna: Presencia de Spartina alterniflora en humedales mexicanos

5. Rizoma y escamas con tejido aerenquimatoso; lemas con ápice usualmente acuminado; plantas nativas de la costa del océano Atlántico (Tamaulipas y Veracruz)

S. alterniflora

Spartina alterniflora Loisel., Fl. Gall. 719. 1807. Fig. 1.

Hierba acuática perenne; rizomas alargados, carnosos. Culmos erectos, de 40 a $250 \mathrm{~cm}$ de longitud; vainas sin quilla; lígula una hilera de pelos de 1 a $1.8 \mathrm{~mm}$ de longitud. Hojas persistentes, lámina de 10 a $40 \mathrm{~cm}$ de largo y de 5 a $10 \mathrm{~mm}$ de ancho; superficie acostillada, ápice acuminado, coriáceo. Inflorescencia una panícula erecta, unilateral, de 10 a $20 \mathrm{~cm}$ de longitud, 5 a 13 racimos digitados o a lo largo de un eje central de 1 a $5 \mathrm{~cm}$ de largo; raquis angular, glabro en los márgenes, terminando en una extensión estéril de 15 a 27 mm de largo. Espiguillas adpresas, solitarias, sésiles, distribuidas a lo largo del raquis en dos hileras; lanceoladas u oblongas, lateralmente comprimidas, de 10 a $15 \mathrm{~mm}$ de largo, uniflosculares, sin extensión de la raquilla, cayendo enteramente; glumas diferentes, excediendo el ápice de los flósculos; gluma inferior linear, 0.66 de lo largo de la gluma superior, membranácea, 1 aquillada, 1 a 3 nervada, escabriúscula, ápice agudo; gluma superior con ápice agudo, superficie glabra; lema fértil ovada, de 8 a $12 \mathrm{~mm}$ de longitud, membranácea, más delgada en los márgenes, aquillada, 1 a 5 nervada, superficie glabra, ápice agudo; pálea de 8.8 a 13 mm de longitud, 2 nervada. Anteras 3, de 5 a 6 mm de longitud, estigmas protóginos, exsertos, pubescentes. Cariopsis linear.

Spartina alterniflora es nativa de América y se distribuye naturalmente en las costas del Océano Atlántico desde Canadá, Estados Unidos de América, Caribe, Guyana, Guyana Francesa, Surinam, Argentina, Uruguay (Peterson et al., 2001) y en México (Tamaulipas y Veracruz). Su hábitat en México son las aguas salobres de estuarios y ríos, muy cerca de la desembocadura con el mar y a profundidades de 20 a $100 \mathrm{~cm}$. Forma densas poblaciones puras o en asociación con Schoenoplectus robustus, Rhizophora mangle, Laguncularia racemosa y/o Avicennia germinans. Su floración se presenta ente los meses de julio a noviembre. De acuerdo con Allan (1950) y Webb et al. (1985), esta hidrófita se reproduce por semillas y rizomas en humedales costeros donde es importante como refugio y alimento de la fauna silvestre. Sin embargo, se comporta como maleza acuática en los sitios donde ha sido introducida, como Asia, Europa, Australia y suroeste de Estados Unidos donde ha desplazado a otras plantas acuáticas nativas (Webber, 2003; Ayres et al., 2004). 


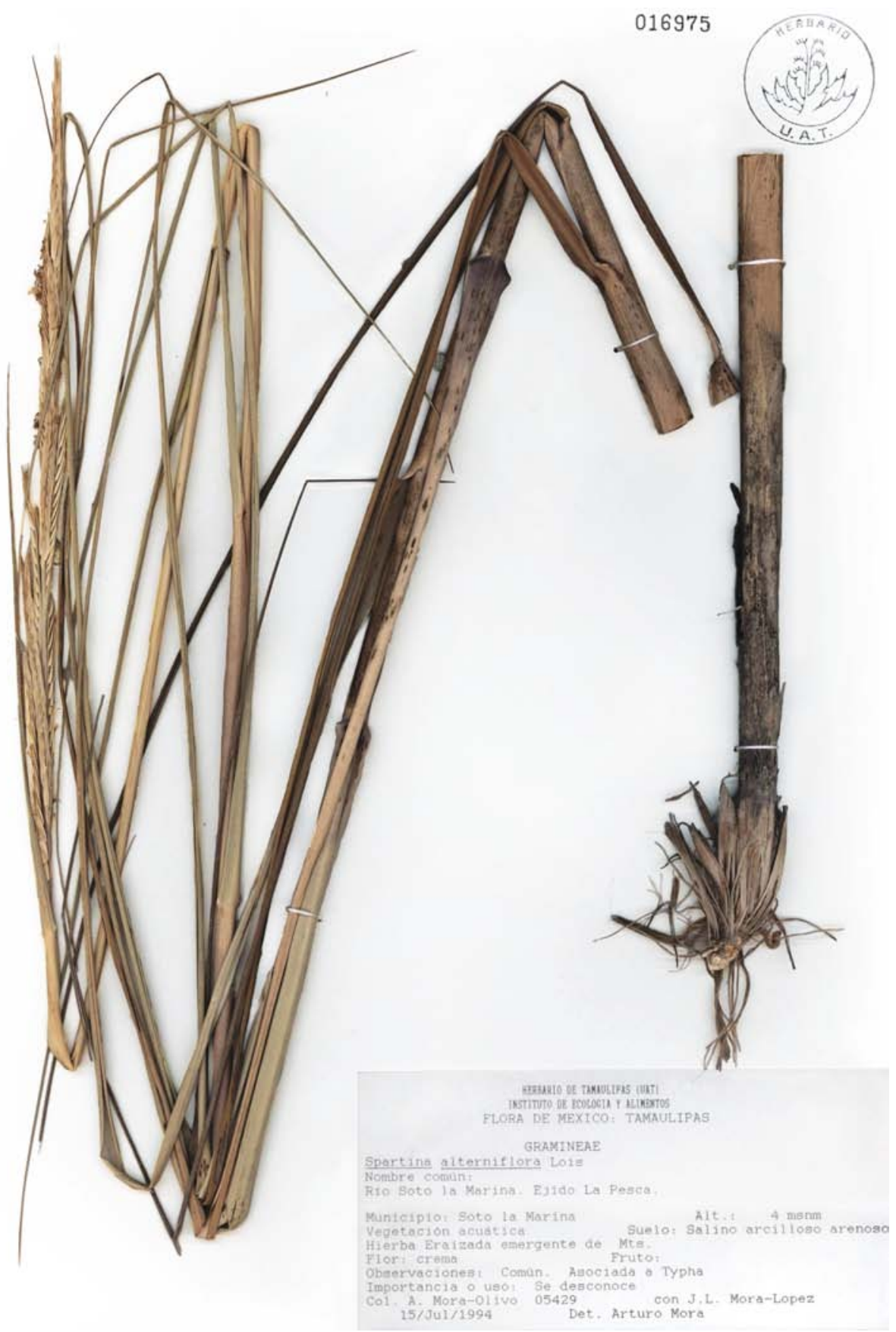

Fig. 1. Ejemplar de Spartina alterniflora colectado en el estado de Tamaulipas. 
Aunque en este trabajo se considera aún a Spartina como parte de la tribu Cynodonteae, recientes estudios filogenéticos apoyan cada vez más la inclusión de este género dentro de Zoysieae y es muy probable que pronto se considere como parte de Sporobolus (Peterson et al., 2010).

Ejemplares examinados: TAMAULIPAS: municipio de Matamoros, río Bravo, Los Chorreaderos, $1.5 \mathrm{~km}$ al SO de la desembocadura del río, $1 \mathrm{~m}, 25^{\circ} 56^{\prime} 50.6^{\prime \prime} \mathrm{N}$, 9709'33.3" W, A. Mora-Olivo 11142 (MEXU, UAT); municipio de Soto la Marina, río Soto la Marina, La Pesca, 4 m, 2348'50.0" N, 97²45'42.0" W, A. Mora-Olivo 5429 (ENCB, MEXU, UAT, XAL); río Soto la Marina, Vista Hermosa, 2 m, 2347'49.0" N, 9754'29.2" W, F. Iribe 514 (MEXU). VERACRUZ: Vega del río Pantepec, 10 m, Brigada Veracruz 206 (MEXU); municipio de Tuxpan, orilla del río Tuxpan, cerca de la Laguna de Tampamachoco, 5 m, 2058'00" N, 97²0'00" W, A Sandoval 403 (XAL).

\section{AGRADECIMIENTOS}

Se agradece a los doctores Adolfo Espejo, Ana Rosa López Ferrari y Teresa Mejía Saulés por su apoyo y disposición para publicar este registro. El primer autor agradece al Programa de Mejoramiento del Profesorado de la Universidad Autónoma de Tamaulipas y al Posgrado en Ciencias Biológicas de la Universidad Nacional Autónoma de México su apoyo para la realización de sus estudios de doctorado, durante los cuales se elaboró parte de este trabajo.

\section{LITERATURA CITADA}

Allan, P. F. 1950. Ecological bases for land use planning in Gulf Coast marshlands. J. Soil and Water Conserv. 5: 57-62.

Ayres, D. R., D. L. Smith, K. Zaremba, S. Klorh y D. R. Strong. 2004. Spread of exotic cordgrassand hybrids (Spartina sp.) in the tidal marsh of San Francisco Bay, California, USA. Biol. Invas. 6: 221-231.

Barkworth, M. E. 2003. Spartina Schreb. In: Barkworth, M. E., K. M. Carpels, S. Long y M. B. Piep (eds.). Flora of North America North of Mexico. Vol. 25. Magnoliophyta: Commelinidae (in part): Poaceae, part 2. Oxford University Press. New York. pp. 240-251.

Clayton, W. D., K. T. Harman y H. Williamson. 2009. GrassBase - The Online World Grass Flora. http://www.kew.org/data/grasses-db.html (acceso el 31 de octubre 2009). 
Dávila, P., M. T. Mejía-Saulés, M. Gómez-Sánchez, J. Valdés-Reyna, J. J. Ortiz, C. Morín, J. Castrejón y A. Ocampo. 2006. Catálogo de gramíneas de México. Universidad Nacional Autónoma de México y Comisión Nacional para el Conocimiento y Uso de la Biodiversidad. México, D.F. 671 pp.

Espejo-Serna, A., A. R. López-Ferrari y J. Valdés-Reyna. 2000. Poaceae Barnhart. In: Espejo-Serna, A. y A. R. López-Ferrari (eds.). Las monocotiledóneas mexicanas. Una sinopsis florística. 1. Lista de referencia. Partes IX a XI Pandanaceae a Zosteraceae. Consejo Nacional de la Flora de México, Universidad Autónoma Metropolitana Iztapalapa y Comisión Nacional para el Conocimiento y Uso de la Biodiversidad. México, D.F. pp. 7-236.

Mobberley, D. G. 1956. Taxonomy and distribution of the genus Spartina. Iowa State College J. Sci. 30: 471-574.

Peterson, P. M. 2001. Spartina. In: Peterson, P. M., R. J. Soreng, G. Davidse, T. S. Filgueiras, F. O. Zuloaga y E. J. Judziewicz (eds.). Catalogue of New World Grasses (Poaceae): II. Subfamily Chloridoideae. Contr. U. S. Natl. Herb. 41: 195-200.

Peterson, P. M., K. Romaschenko y G. Johnson. 2010. A classification of the Chloridoideae (Poaceae) based on multi-gene phylogenetic trees. Mol. Phylogenet. Evol. 55: 580598.

Webb, J. W., S. K. Alexander y J. K. Winters. 1985. Effects of autumn application of oil on Spartina alterniflora in a Texas salt marsh. Env. Poll. 38(4): 321-337.

Webber, E. 2003. Invasive plant species of the world: a reference guide to environmental weeds. CAB Publishing. Wallingford, U.K. 560 pp. 